\title{
Modeling Growth Kinetics of Methane Hydrate in Stirred Tank Batch Reactors
}

\author{
Divya Gootam ${ }^{1}$, Namrata Gaikwad ${ }^{1}$, Rajnish Kumar ${ }^{*}$, Niket Kaisare ${ }^{1 *}$ \\ ${ }^{1}$ Department of Chemical Engineering, Indian Institute of Technology Madras, \\ Chennai 600036, India
}

*Corresponding author(s):

1. Rajnish Kumar: email: rajnish@iitm.ac.in.

2. Niket Kaisare: e-mail: nkaisare@iitm.ac.in.

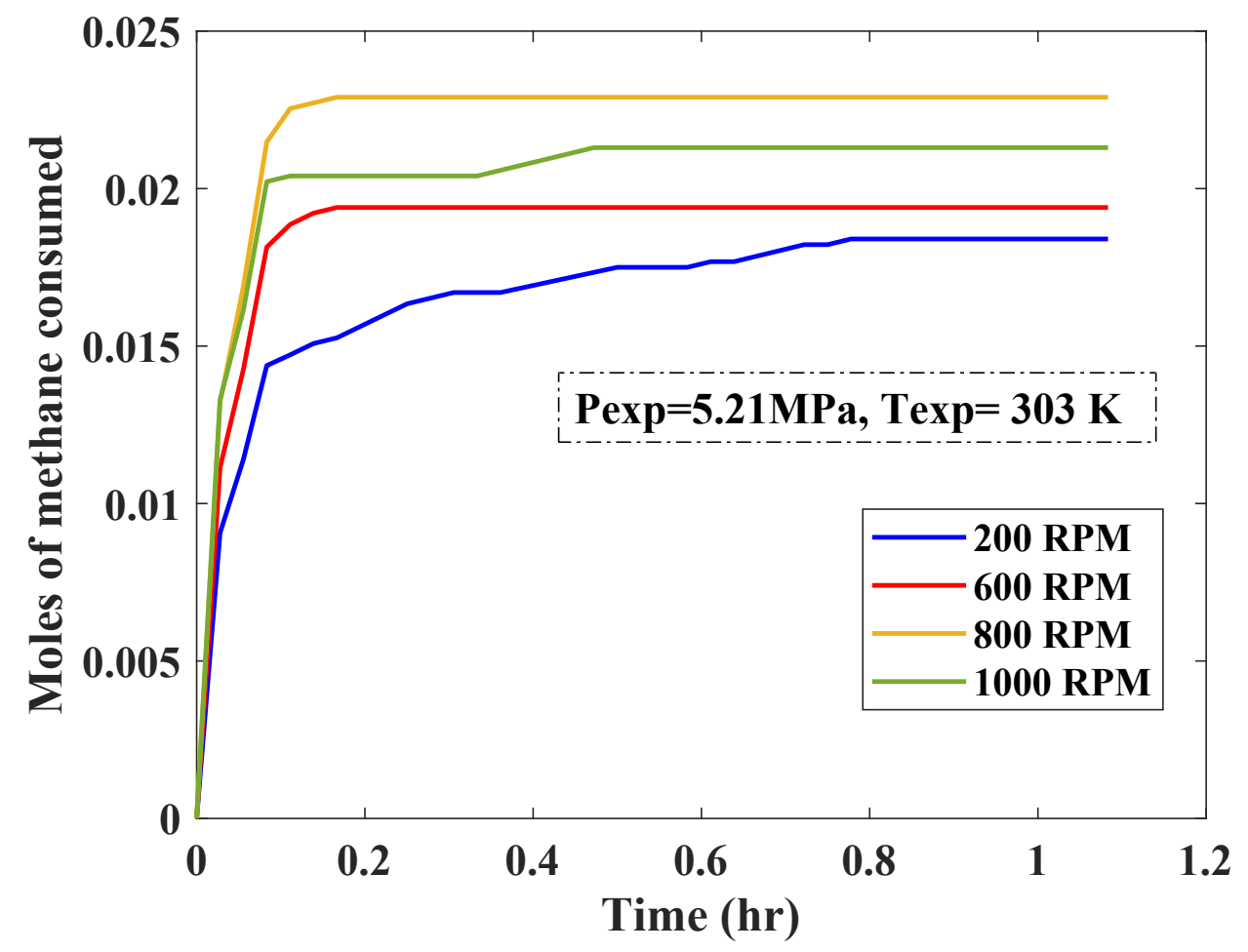

Figure S1: Effect of stirrer speed on methane dissolution in water at 5.21 MPa and $303 \mathrm{~K}$. 

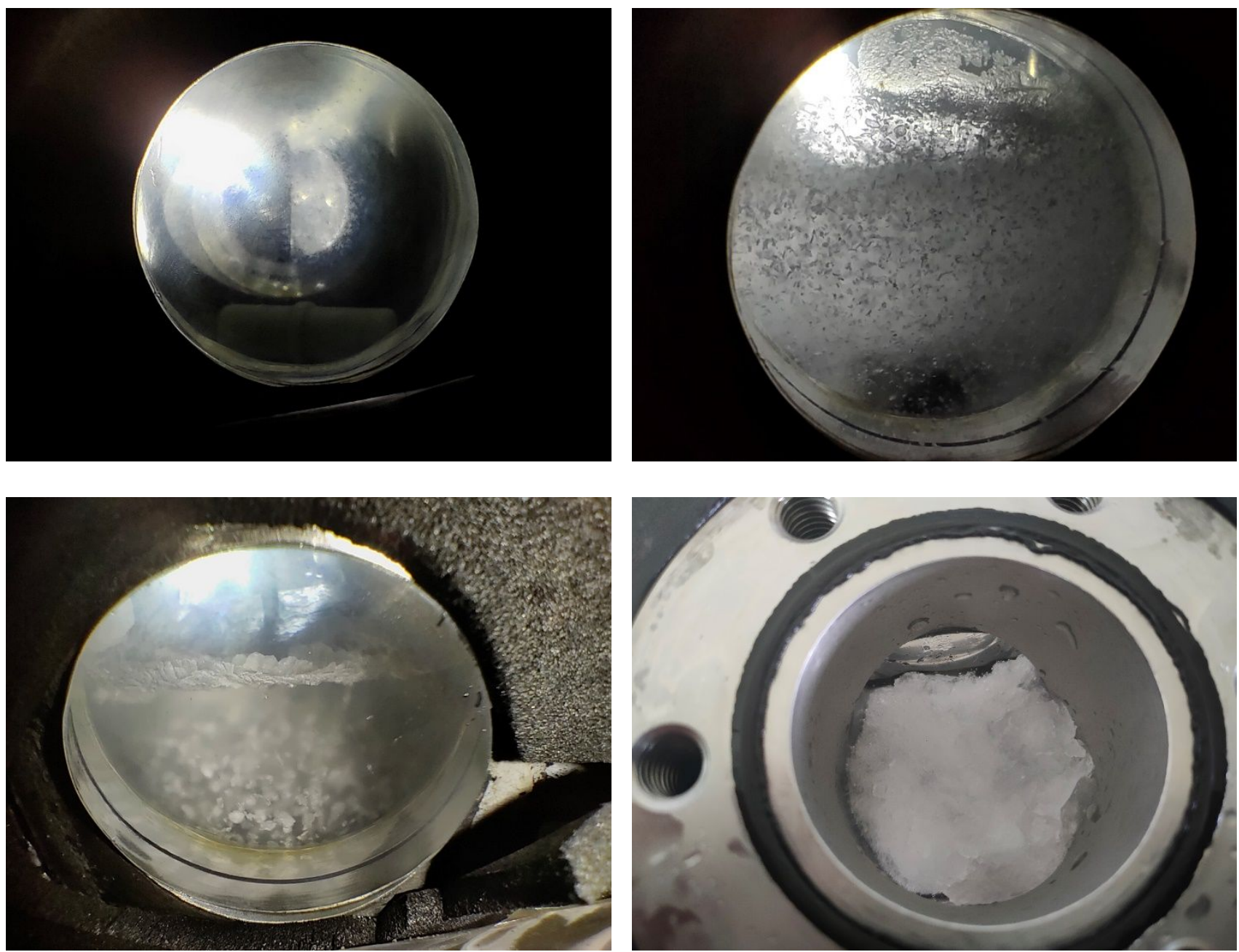

Figure S2: Morphology of methane-water

reaction (a) At turbidity point (b) Few minutes after reaching turbidity point (c) At the end of the reaction (i.e.) no further pressure drop (d) After depressurising the reaction. 

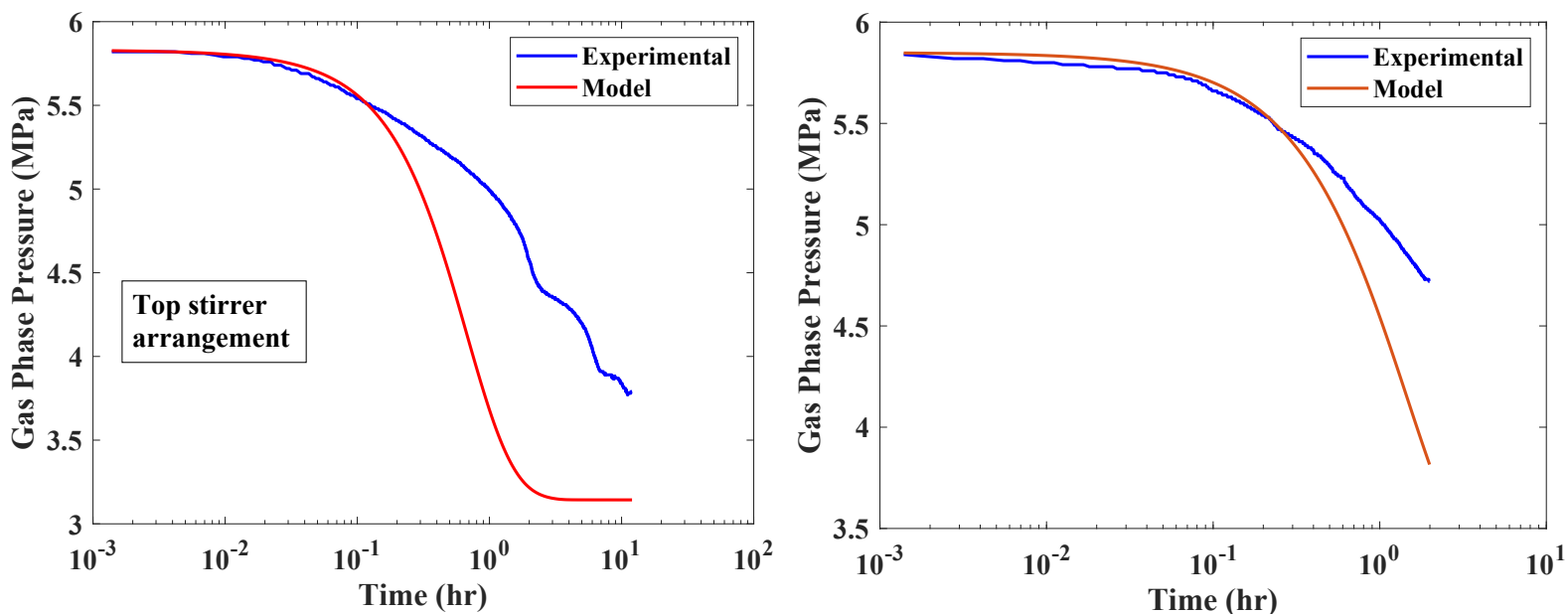

Figure S3: Semi-log plots of top and bottom stirrer arrangements. 